\title{
105
}

\section{Robotics: An Agent of Change for ICT?}

\author{
Chair: Erling Schmidt (DK) \\ Panel: Tom Lough (US), Cathy Fett (US)
}

Keywords: robotics, innovation, equality issues, collaborative learning, programming

This panel was designed to stimulate a discussion of robotics as an agent of change in education. The initial remarks by the panel were based on a survey mailed out to (US) schools early in 2001. The following questions were discussed. Do robotics activities affect male students differently than female students? What are some of the affective aspects of robotics activities? Do robotics in schools seem to have any impact on student career choices?

The 165 survey respondents were almost equally male and female, and were evenly distributed in teaching experience. The most common grades for robotics were in the middle school range.

The patterns for the responses describing male and female students were similar for several items. Male and female students were reported mainly 'quite often' for (a) demonstrates on-task focus of long duration, (b) collaborates with other students of the same gender, and (c) expresses interest and excitement. Male and female students were reported mainly 'somewhat often' for (a) demonstrates frequent and familiar use of robotics terms in operational vocabulary, (b) shows an emotional connection with robotics projects, (c) shows evidence of having discussed robotics with parents or other family members, (d) demonstrates uncommon persistence in the face of a daunting problem, and (e) participates in other technologyrelated programs (in school or outside of school).

Patterns for the responses describing male and females students were different for several items. Female students were described as having higher levels of the following activities than male students: collaborates with 
students of the opposite gender, accepts role willingly in teamwork situations, and shows a desire to work with others. Male students were described as having higher levels of the following activities than female students: tends to work by oneself, shows evidence of unprompted or nonrequired robotics work, spontaneously asks questions about other robotics applications, is willing to design and build a rough prototype that may or may not work, and comes to class on 'free time' to spend more time with robotics.

As observed by respondents, the vast majority of students want to participate in robotics activities, reporting for males very much $73 \%$ and somewhat $23 \%$, and for females very much $36 \%$ and somewhat $41 \%$. The responses suggest that female students are perceived to be more interested in collaboration and may be better team players than male students. Yet, male students seem to 'get into' robotics more than the female students.

Responses in the career-related items indicated little activity. Several of the items related more to students of high school age than to younger students.

Ideas and suggestions for teachers included (a) provide a combination of collaborative and competitive activities to appeal to both genders, (b) try different group combinations to adjust the social dynamics, (c) be aware of the attention given to the different groups, (d) make an effort to engage girls in the process so they participate more fully, and (e) allow some creative freedom in building and programming levels so all students can build and/or program to his or her highest level.

Panel attendance was satisfactory. A few questions were asked by members of the audience, and there were some interactions among audience members. 